\title{
ETNOINFORMÁTICA NA EDUCAÇÃO: Uso N'SAMAT como organizador prévio para auxiliar na aprendizagem de Aritmética em três escolas moçambicanas.
}

\author{
Florêncio Extermo Maulano - PPGIE/UFRGS, fmaulano1@gmail.com \\ Rosa Maria Vicari - PPGIE \& PPGC/UFRGS, rosa@inf.ufrgs.br \\ Maria Cristina Villanova Biasuz - PPGIE/UFRGS, mcbiazus@gmail.com
}

Resumo: O presente artigo propõe o uso do N'SAMAT, em aula, para auxiliar na melhoria do aproveitamento dos alunos da $2^{\mathrm{a}}$ classe do ensino básico na aprendizagem de Aritmética (o que corresponde ao ensino fundamental series iniciais, no Brasil). Para concretização do estudo, foi adotado um plano quase-experimental, associado a uma pesquisa quali-quantitativa, em que foi feito um estudo inicial junto aos gestores e professores e a posteriori uma avaliação do experimento com os alunos, na qual foram submetidos a um pré-teste e a um pós-teste. O pré-teste consistiu na resolução de uma prova de matemática no primeiro momento, já no segundo momento os alunos tiveram uma intervenção com auxílio do objeto de aprendizagem N'SAMAT e culminou com a resolução do pós-teste. Dos resultados obtidos conclui-se que a metodologia aplicada contribuiu para o aproveitamento dos alunos na disciplina de matemática, especificamente, em conteúdos ligados à aritmética.

Palavras-Chave: Aproveitamento escolar, Aprendizagem significativa, Jogo tradicional, Objeto de aprendizagem, N’SAMAT.

\section{ETNOINFORMATICS IN EDUCATION: Use N'SAMAT as a previous organizer to assist in learning Arithmetic in three Mozambican schools.}

Abstract: The present article proposes the use of N'SAMAT, in class, to assist in the improvement of the use of the students of the 2nd class of basic education in learning Arithmetic. To carry out the study, a quasi-experimental plan was adopted, associated with a qualitative-quantitative research, in which an initial study was carried out together with the managers and teachers and a posteriori evaluation of the experiment with the students, in which they were submitted to a pre-test and a posttest. The pre-test consisted of solving a math test in the first moment, and in the second moment the students had an intervention with the aid of the N'SAMAT learning object and culminated in the resolution of the post-test. From the results obtained, it is concluded that the applied methodology contributed significantly to the students' achievement in the mathematics discipline, specifically in content related to arithmetic.

Key words: School achievement, Significant learning, Traditional game, Learning object, N'SAMAT.

\section{Introdução}

A expansão tecnológica e a expansão da rede escolar precisam ocorrer levando em conta o contexto e o ambiente do aluno nos dias de hoje, de modo que o seu contexto sociocultural seja valorizado e aprimorando. Para tal, tanto a tecnologia quanto a cultura, que atualmente desempenham um papel muito importante no desenvolvimento de um País devem andar juntas. E tal desenvolvimento precisa estar à mercê de indivíduos com uma boa capacidade criativa e inovadora que evoluam no mesmo ritmo que os avanços tecnológicos, mas sem perder a sua identidade, fazendo com que as novas descobertas sejam um meio para o desenvolvimento de sua própria cultura. Portanto, a etnoinformática pode ser o processo pelo qual, existe a reutilização de aspectos 
socioculturais de um povo ou comunidade, ao longo do tempo, sejam esses, jogos, danças, músicas entre outras artes para o desenvolvimento de objetos de aprendizagem ou softwares educacionais na era digital.

Este artigo tem como objetivo, apresentar a utilização do objeto de aprendizagem N'SAMAT, em aula, com a finalidade de auxiliar no melhoramento do aproveitamento dos alunos da $2^{\text {a }}$ classe do ensino básico, na aprendizagem de Aritmética, em Moçambique. Tal desafio teve como foco a integração de jogos tradicionais apoiados nas tecnologias: "Objeto de aprendizagem culturalmente contextualizado N'SAMAT”, em ambientes escolares para responder às dificuldades que os alunos enfrentam em preservar aspectos socioculturais, bem como a falta de motivação na aprendizagem de cálculo no ensino fundamental.

Neste contexto, o N'SAMAT mostrou alcançar uma abrangência tanto cultural como tecnológica em sala de aula. Com isso, a ideia de aplicar o N'SAMAT pode ser uma boa alternativa para a aprendizagem da aritmética, simultaneamente, o mesmo contribui para o reencontro entre os alunos da zona rural e as novas tecnologias de informação e comunicação, do mesmo modo que contribui para reaproximação dos da zona urbana com valores socioculturais que foram se deteriorando ao longo dos anos pela desconexão com a sua origem e a fragmentação de sua cultura ao longo do tempo.

\section{Objeto de aprendizagem}

Na óptica de (WILEY, 2000 apud TAROUCO et al 2014, p. 13) um objeto de aprendizagem: "[...] é qualquer recurso digital que pode ser reusado para apoiar a aprendizagem". Essa definição incorpora as palavras: "reusado”, “digital”, “recurso”, “aprendizagem”, conforme especifica o Comitê de Padrão de Tecnologia da Aprendizagem (Learning Technology Standard Committee LTSC).

Tarouco et al (2014) afirma que, Wiley (2002) explica que um objeto de aprendizagem utiliza a metáfora de um átomo, ou seja, um elemento pequeno que pode ser combinado e recombinado com outros elementos, formando algo maior. Portanto, a autora define objetos de aprendizagem como sendo qualquer recurso, suplementar ao processo de aprendizagem, que pode ser reusado para apoiar a aprendizagem, termo geralmente aplicado a materiais educacionais projetados e construídos em pequenos conjuntos visando a potencializar o processo de aprendizagem onde o recurso pode ser utilizado.

\subsection{Aprendizagem significativa e Objeto de aprendizagem.}

Os alunos possuem conhecimento prévio, cabe ao professor estimulá-lo de modo que esse possa ser usado para auxiliar na construção do novo conhecimento. Mas, para que tal aconteça, o professor precisa usar estratégias pedagógicas que estimulem, passando a recorrer a organizador prévio. Para Ausubel, Novak e Hanesian (1980), organizadores prévios são materiais introdutórios apresentados antes do próprio material a ser aprendido. Ausubel, Novak e Hanesian (1980) ressaltam a necessidade da predisposição para aprendizagem significativa. O aluno tem papel crucial para ocorrência desta, visto que uma tarefa pode ser significativa para determinados alunos e mecânica 
para outros, dependendo dos conhecimentos prévios que estes apresentem. Às vezes, o aluno não está familiarizado com o assunto em questão e pode utilizar a estratégia de internalizar a atividade de forma arbitrária, decorando literalmente as orientações que lhe foram apresentadas pelo professor. Sendo assim acreditamos que o envolvimento dos alunos em atividades estruturadas durante o período na qual foi aplicado a metodologia usando materiais manipuláveis, neste caso o objeto de aprendizagem N'SAMAT em sala de aula, na disciplina de matemática permitiu aos alunos descobrirem relações, fazerem reflexões, bem como construírem conceitos que poderão lhes auxiliar na compreensão de conceitos, tais como:

- Adição e subtração de números naturais;

- Contagem de números naturais até 10;

- Ordenação de números naturais de ordem crescente;

No entanto, a preposição na implementação do objeto de aprendizagem N'SAMAT é que os alunos pudessem assimilar significativamente os conceitos de adição e subtração, contagem e ordenação de números naturais a serem estudados, se estes descobrirem o encadeamento lógico na construção do conhecimento matemático. A Teoria da Aprendizagem Significativa apresenta alguns princípios característicos que podem favorecer a aquisição do conhecimento escolar. Ausubel (2002) propõe que os alunos podem realizar aprendizagem significativa dos conceitos estudados, quando os mesmos estiverem organizados segundo uma sequência lógica, denominada diferenciação progressiva. Durante o desenvolvimento das ações contidas nas tarefas potencialmente significativas, o que provavelmente facilitará a assimilação dos conceitos são as atividades que servirão como base para a construção do conhecimento, o que foi denominado por Ausubel de organizadores prévios.

Desse modo, o uso do objeto de aprendizagem N'SAMAT em sala de aula apresenta-se como uma estratégia pedagógica dinâmica e motivadora para a compreensão dos conceitos de adição e subtração, contagem e ordenação de números naturais, de modo que a aprendizagem mostra um processo próprio da interação do aluno com o meio, permitindo uma mudança afincada no seu potencial. Dessa maneira, o objeto de aprendizagem N'SAMAT possui essa qualidade na interação e na valorização dos saberes dos sujeitos (vide a Imagem 1).

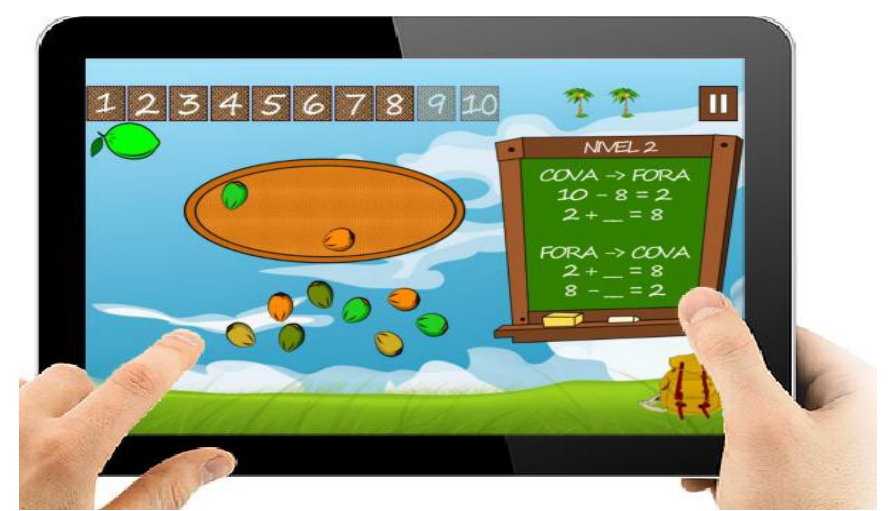

Imagem 1: Ambiente de Jogo do OA N'SAMAT

Fonte: Adaptado (MAULANO e MATHEUS, 2016). 
Segundo Vasconcelos e Lima (2012), pode-se afirmar que, no caso da matemática, é possível organizar essas informações com o auxílio do uso de jogos, de variadas maneiras, de forma que a escolha e planejamentos coerentes impliquem em atribuições cognitivas positivas, evitando assim, uma sobrecarga de informações do ponto de vista de promover uma aprendizagem significativa.

Para Ausubel (2002), aprender significativamente é transformar um conjunto de informações (conteúdos e procedimentos) em algo útil para a vida. Ele assinala que para existir uma aprendizagem significativa, deve haver a preexistência de significados e o material utilizado pelo professor seja compatível com a estrutura cognitiva do aluno, isto ocorrendo de maneira natural, ou seja, não arbitrária. O material ou organizador prévio utilizado para esse fim deve ser potencialmente significativo.

Quando os conceitos subsunçores ${ }^{1}$ são pouco elaborados ou inexistentes, cabe ao professor utilizar-se dos organizadores prévios, que servem de ponte entre o que o aprendiz já sabe e o que ele vai aprender.

Esses organizadores servem de âncora para a nova aprendizagem e são os facilitadores da aprendizagem subsequente. Podem ser representados por uma pergunta, um problema, um filme um texto, materiais manipuláveis etc.

\subsection{N'SAMAT como organizador prévio}

Nunes, et al (2010) diz que, caso os conceitos relevantes não estiverem disponíveis na estrutura cognitiva, o organizador prévio servirá para ancorar novas aprendizagens e levar ao desenvolvimento de um conceito subsunçor que facilitasse a aprendizagem subsequente.

Desse modo, achamos que o objeto de aprendizagem N'SAMAT pode ser um recurso facilitador de uma aprendizagem significativa, desde que se habilite como organizador prévio.

O N'SAMAT apresenta-se como um objeto de aprendizagem para auxiliar na assimilação significativa dos conceitos de adição, subtração, contagem e ordenação de números naturais, evidenciando a construção de ideias que contribuem para o desenvolvimento destes. Portanto, o uso de objeto de aprendizagem N'SAMAT como material paradidático pode ajudar na compreensão de conceitos, habilitando esses a organizadores prévios.

Neste caso, os conceitos matemáticos não são criados arbitrariamente, eles surgem da ação sobre outros já existentes, que, por sua vez, foram criados para atender as necessidades de atividades cotidianas. Portanto, a ideia de adotar o objeto de aprendizagem N'SAMAT como organizador prévio é mostrar que o aluno pode aprender aritmética como base em situações do seu cotidiano, através de atividades lúdicas “Jogos tradicionais” associadas as tecnologias digitais (vide a imagem 3).

1 Ausubel define este conhecimento prévio como "conceito subsunçor" ou simplesmente "subsunçor". Os subsunçores são estruturas de conhecimento específico que podem ser mais ou menos abrangentes de acordo com a frequência com que ocorre aprendizagem significativa em conjunto com um dado subsunçor. 


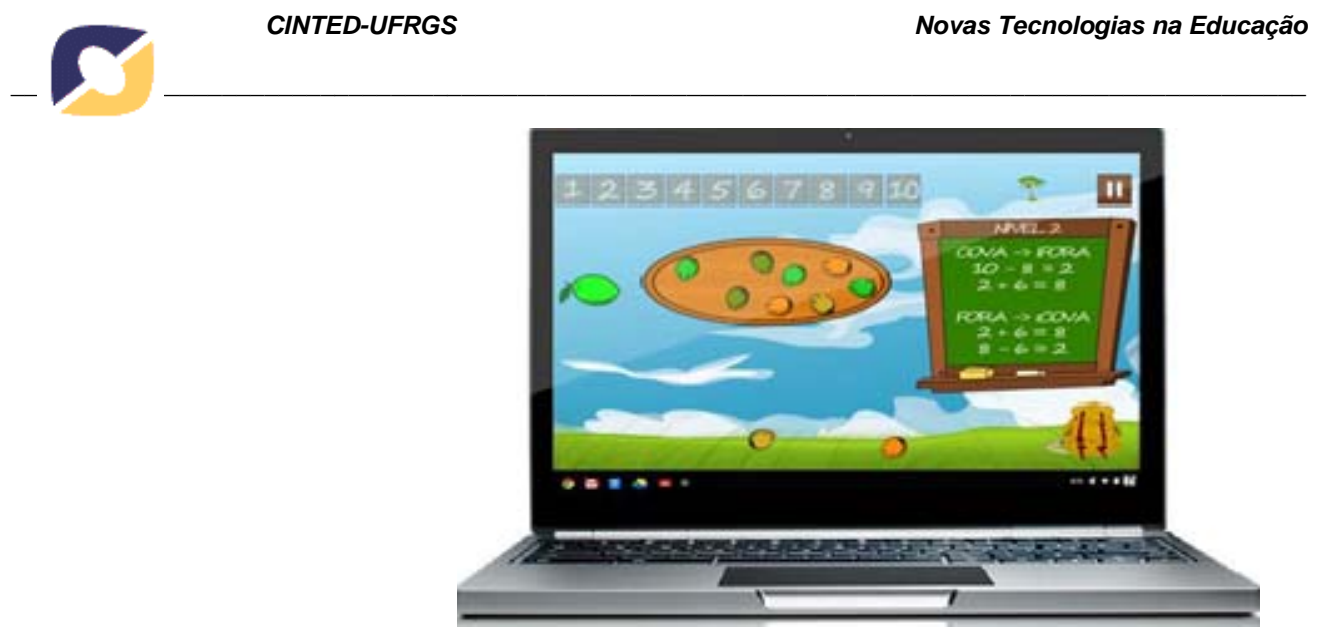

Imagem 3: Ambiente de Jogo do OA N'SAMAT

Fonte: Adaptado (MAULANO e MATHEUS, 2016)

De acordo com as descrições feitas anteriormente, a metodologia aplicada com auxílio do objeto de aprendizagem N'SAMAT, visa contribuir para compreensão de noções de conteúdos matemáticas à luz da teoria da aprendizagem significativa. Propondo a necessidade de se trabalhar com os alunos, em atividades que os coloquem em contato com a construção das ideias matemáticas a partir dos seus conhecimentos prévios. Ou seja, a aprendizagem significativa consiste em relacionar, de forma não arbitrária e substantiva (não ao pé da letra), uma nova informação a outra com as quais o aluno já esteja familiarizado. (AUSUBEL, 1976)

\section{Metodologia}

O artigo contempla uma pesquisa com perspectiva quanti-qualitativa a partir do plano quase experimental. Paralelamente ao plano de pesquisa quase experimental, é aplicado o estudo com o desenho pré e pós-teste em dois grupos, onde um recebe tratamento experimental e o outro serve de grupo de controle. Esta conjugação de metodologias surge com o objetivo de proporcionar uma maior familiarização do assunto estudado ao pesquisador, visto que no decorrer das atividades este atua com coordenador do grupo e que a atividade em causa tem poucos estudos, sendo assim pouco explorado na atualidade.

Foi feita uma análise e validação dos resultados do experimento final, baseando-se na comparação dos experimentos entre si, bem como com outras pesquisas realizadas e publicadas nesta área. Para a realização da análise de dados, foi usado o método estatístico compatível com os procedimentos metodológicos propostos. Essa análise foi feita com base no software SAS. A unidade experimental foi composta por 60 alunos, do $2^{\mathrm{a}}$ ano do ensino fundamental, de três escolas selecionadas para participar do estudo, na Província da Zambézia, em Moçambique. O fator de interesse é o tempo transcorrido entre as duas provas, tendo a exposição ao jogo entre elas, gerando dois níveis: antes da exposição ao jogo e após a exposição ao jogo.

A variável resposta que mede o efeito do fator tempo, isto é, mede o efeito da exposição ao jogo, é a nota das provas aos quais os alunos foram submetidos (medida de desempenho dos alunos). V. $16 \mathrm{~N}^{\circ}$ 1, julho, 2018 
O modelo ajustado foi o de análise de variância para medidas repetidas (dados correlacionados) via modelos mistos, utilizando o software SAS STUDIO 2. Supondo que o aproveitamento dos alunos (nota das provas) possa ser afetado pela zona de origem da escola (urbana ou rural), sexo e idade dos alunos, estas três variáveis foram utilizadas como ajuste no modelo.

\section{Resultados}

O objetivo do estudo é compreender a influência do N'SAMAT na aprendizagem da Aritmética, para tal, os alunos foram submetidos a um teste antes (Pré-teste - “1”) e outro depois (Pós - Teste - “2”). O facto de termos dados quantitativos organizados em dois grupos nos traz a possibilidade de aplicar um teste $\mathrm{T}$ de Student, adicionalmente, o facto de o grupo de indivíduos submetidos no primeiro e segundo teste ser o mesmo, o mais adequado é um teste T de Student com medidas repetidas ou Teste $\mathrm{T}$ : duas amostras emparelhadas para médias.

Tabela 1. Variável de análise: NOTA

\begin{tabular}{r|r|r|r|r|r|r}
\hline \multicolumn{7}{c}{ Analysis Variable : NOTA NOTA } \\
\hline TEMPO & $\begin{array}{r}\text { N } \\
\text { Obs }\end{array}$ & $\mathbf{N}$ & Mean & Std Dev & Minimum & Maximum \\
\hline 1 & 60 & 60 & 7.4566667 & 3.6644128 & 1.0000000 & 15.0000000 \\
\hline 2 & 60 & 60 & 12.5166667 & 3.8894977 & 5.0000000 & 20.0000000 \\
\hline
\end{tabular}

Fonte: Adaptado pelo pesquisador [NAE, 2018]

A Tabela 1. Ilustra a estatística descritiva, na qual põe em evidência os dois momentos do pré-teste e pós-teste. No primeiro momento foram feitas 60 observações que corresponde ao número de alunos distribuídos em três escolas respectivamente, sendo duas escolas da zona urbana e uma da zona rural, em que as suas notas variam de 1 a 15 valores, correspondendo a uma média de aproximadamente 7.5 valores e um desvio padrão de 3.7 .

Já no segundo momento, o cenário se repete quanto ao número de observações. Ao serem observados 60 alunos cuja a sua distribuição também se caracterizou na zona urbana e rural, respectivamente, e em três escolas sendo uma ao nível do distrito e duas ao nível da cidade. A Tabela 1 mostra que as suas notas variam de 5 a 20 valores (em Moçambique as notas variam entre 0 e 20 valores), com uma média de aproximadamente 12.5 valores e um desvio padrão rondando nos 3.9, o que mostra no primeiro instante que a média do segundo teste é significativamente superior que a do primeiro teste. Pode-se concluir que:

O aluno aprende significativamente Matemática, quando consegue atribuir sentido e significado às ideias matemáticas - mesmo aquelas mais puras (isto é, abstraídas de uma realidade mais concreta) - e, sobre elas, é capaz de pensar, estabelecer relações, justificar, analisar, discutir e criar. (FIORENTINI, 1995, p.32)

Associado a essa ideia, se pode dizer que o conhecimento matemático deve proporcionar condições para que o aluno possa inteirar-se acerca de questões culturais, sociais e históricas que vive 
no seu dia a dia. Dessa forma, a falta de conexão entre a matemática escolar e a matemática da vida cotidiana do aluno, bem como a não diversificação de estratégias em sala de aula, são fatores que contribuem para as dificuldades que os alunos enfrentam na compreensão de determinados conteúdos no contexto escolar.

\subsection{Análise exploratória}

Verificando a média das notas por zona (gráfico 1), nota-se claramente a diferença entre os alunos da zona rural e da zona urbana. Os alunos da zona 1 (urbana) tem nota média superior aos alunos da zona 2 (rural), independentemente do tempo de exposição ao jogo.

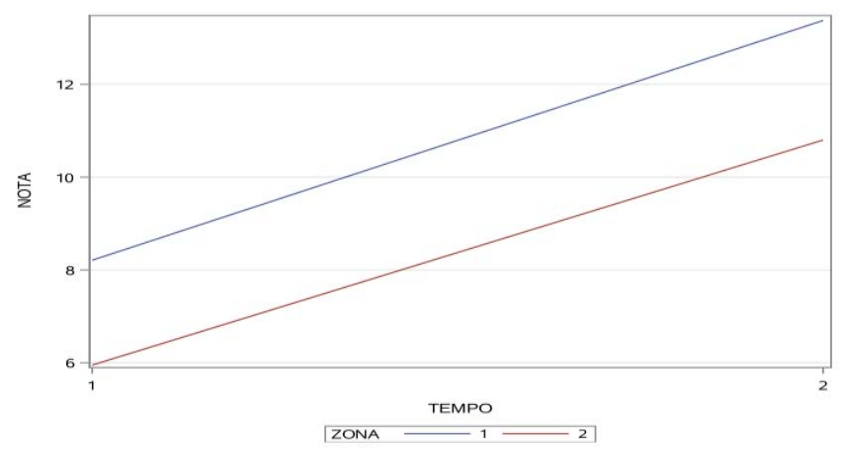

Gráfico 1: Notas médias por zona de origem da escola dos alunos

Fonte: Adaptado pelo pesquisador [NAE, 2018]

O estudo faz referência a distribuição da amostra, tendo em conta a zona de origem de alguns participantes, essa escolha deve-se ao fato de se tratar de duas realidades completamente diferente, o que gerou uma análise tendo em conta a variável zona conforme o gráfico 1. Este gráfico ilustra que a média dos testes realizados na zona urbana são superiores relativamente as médias dos testes realizados na zona rural. Isso deve-se a dois aspectos a elencar:

- Os alunos da zona rural tiveram um aproveitamento da média dos testes relativamente baixo comparado aos alunos da zona urbana, provavelmente devido ao fraco acesso as tecnologias e as infraestruturas escolares precárias;

- Já na zona urbana, o cenário foi diferente, os alunos tiveram um aproveitamento da média dos testes acima da zona rural, devido a facilidade de acesso as tecnologias, infraestruturas adequadas com equipamentos informáticos.

Nesta mesma linha, Castro (2000), analisando as diferenças regionais no sistema de ensino brasileiro, revela uma disparidade em termos de qualidade e oferta de serviços educacionais entre as regiões Nordeste e Sudeste, o que resulta numa diferença na média de anos de estudo desfavorável à primeira. Em relação às características individuais e familiares, o fato de o aluno da zona urbana ser do sexo masculino afeta positivamente o desempenho escolar, em Matemática, enquanto na zona 
rural as mulheres têm obtido melhores notas.

Essa constatação, fez concluir que o aproveitamento escolar em zona urbana acaba sendo melhor relativamente a zona rural como ilustra o (gráfico 1).

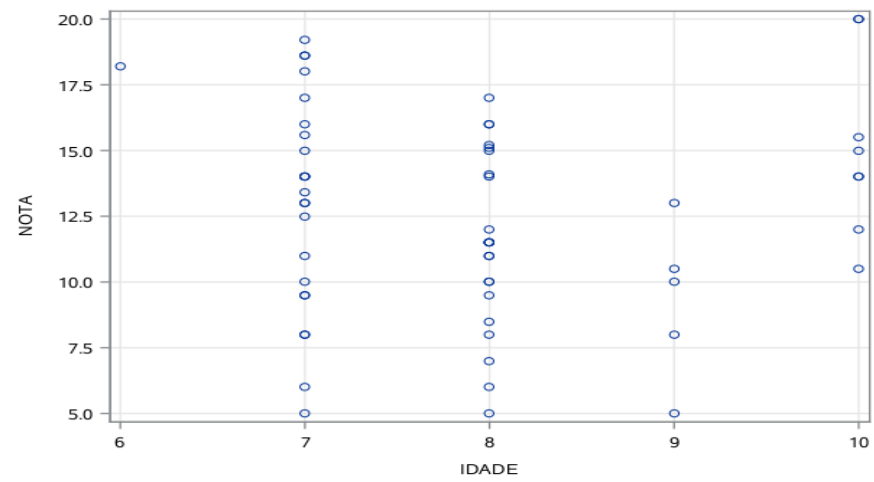

Gráfico 2: Notas médias por Idade dos alunos no pós-teste (T2)

Fonte: Adaptado pelo pesquisador [NAE, 2018]

O mesmo, se pode verificar no gráfico 2, as idades variam de 6 anos a 10 anos, com maior destaque aos alunos com 7 anos de idade na sua maioria, uma vez que se trata da mesma amostra, mas com intervenção em momentos diferente. Neste caso se pode observar que as notas melhoraram significativamente, sendo a maior nota verificada para os alunos com idade compreendida a 10 anos. Ainda, para (BEZERRA e KASSOUF, 2006), esses autores trazem-nos no seu estudo uma situação muito interessante no que diz respeito à idade, afirmando que:

\begin{abstract}
A média é maior para os alunos das áreas rurais. Os alunos das áreas urbanas estão em média com quase um ano acima da idade correta para a série, enquanto nas escolas rurais esse valor é próximo de um ano e meio. De acordo com o sistema educacional do país, um aluno que inicia o curso fundamental aos sete anos e atinge a 4a série sem ter atrasado nenhum ano de seus estudos, estaria, normalmente, com 10 anos. (BEZERRA e KASSOUF; 2006, p. 9)
\end{abstract}

\title{
4.2. Ajuste do modelo
}

Durante aproximadamente quatro semanas, os alunos estiveram expostos, durante 15 a 20 minutos, a metodologia aplicada consistia em usar o objeto de aprendizagem N'SAMAT, em aula, para a melhoria do aproveitamento dos alunos da $2^{\mathrm{a}}$ classe do ensino básico na aprendizagem de Aritmética. Daí que, após ser adotado o modelo ajustado, chegou-se aos seguintes resultados:

Os dados evidenciam que existe efeito da exposição ao jogo (Tempo) sobre o desempenho médios de todos os alunos, independente de zona de origem, de sexo e da idade (Valor $\mathrm{p}<0,0001$ Tabela 1). Ou seja, pode-se afirmar que a metodologia aplicada surtiu resultados desejados, o que remete a ideia de que aplicar o N'SAMAT pode ser uma boa alternativa para a aprendizagem da aritmética.

Associado a teoria de Ausubel, a proposta metodológica da Modelagem Matemática "tem como pressuposto que o ensino e a aprendizagem da Matemática podem ser potencializadas ao se 
problematizar situações do cotidiano. Daí que: “Ao mesmo tempo em que se propõe a valorização do aluno no contexto social, procura levantar problemas que sugerem questionamentos sobre situações de vida” (SEED, 2006, p.6).

Tabela 2: Testes de Tipo 3 de Efeitos Fixos

\begin{tabular}{l|r|r|r|r}
\hline \multicolumn{5}{c}{ Type 3 Tests of Fixed Effects } \\
\hline Effect & $\begin{array}{r}\text { Num } \\
\text { DF }\end{array}$ & $\begin{array}{r}\text { Den } \\
\text { DF }\end{array}$ & F Value & Pr > F \\
\hline ZONA & 1 & 56 & 12.69 & 0.0008 \\
\hline SEXO & 1 & 56 & 1.66 & 0.2023 \\
\hline TEMPO & 1 & 56 & 125.32 & $<.0001$ \\
\hline IDADE & 1 & 56 & 1.89 & 0.1744 \\
\hline
\end{tabular}

Fonte: Adaptado pelo pesquisador [NAE, 2018]

A nota média dos alunos após a exposição ao jogo (12,0620 - Tabela 3) é significativamente maior que a nota média dos alunos antes da exposição ao jogo (7,0042 - Tabela 3). Este aumento médio da nota (aproximadamente 5 pontos em média) ocorreu nas duas zonas de interesse, isto é, tanto na zona urbana como na zona rural.

Segundo (BEZERRA e KASSOUF, 2006), os alunos avaliados em Matemática nas escolas urbanas da região Sudeste tinham um ganho de desempenho de até 16 pontos, sendo para os da zona rural um aumento de 27 pontos, em comparação aos alunos da região Nordeste.

Tabela 3: Notas médias ajustadas dos alunos nas provas aplicadas antes e depois da exposição ao objeto de aprendizagem N'SAMAT.

\begin{tabular}{l|l|r|r|r|r|r}
\hline \multicolumn{7}{c}{ Least Squares Means } \\
\hline Effect & TEMPO & Estimate & $\begin{array}{r}\text { Standard } \\
\text { Error }\end{array}$ & DF & $t$ Value & Pr $>|t|$ \\
\hline TEMPO & 1 & 7.0042 & 0.5044 & 56 & 13.89 & $<.0001$ \\
\hline TEMPO & 2 & 12.0620 & 0.5081 & 56 & 23.74 & $<.0001$ \\
\hline
\end{tabular}

Fonte: Notas médias ajustadas dos alunos

Os dados também evidenciam que existe efeito da zona de origem, isto é, a nota média dos alunos da zona urbana foi significativamente diferente da nota média dos alunos da zona rural ( $\mathrm{p}=0,0008-$ Tabela 2$)$.

Já as variáveis sexo e idade, como sugerido na análise exploratória, não impactaram de forma significativa no ajuste do modelo (seus p-valores são maiores que 0.1 ).

Contudo, foram observados alguns aspectos a ter em conta que estão associados a análise dos resultados a destacar:

- Acessibilidade do Objeto de aprendizagem N'SAMAT, ajudou muito na familiarização dos 
alunos com o jogo, tornando fácil de compreender e manusear;

- Os alunos após se familiarizarem com o ambiente do jogo, foram se interessando por outros aspectos do objeto de aprendizagem N'SAMAT. Tais como: aumentar o grau de dificuldade e passar automaticamente para níveis subsequentes como forma de torná-lo mais desafiante;

- Diante do computador os mais familiarizados com a informática, após terminarem todos níveis do N'SAMAT, iam procurando mecanismos a fim de ascender a outros jogos associados aos jogos digitais que tem vindo a praticar no dia-a-dia.

Entretanto, percebe-se que o jogo é muito simples no seu manuseamento, o que faz com que os alunos não façam muito esforço cognitivo em poder compreendê-lo. Por outro lado, a sua simplicidade se verifica pelo fato do mesmo ter características e origens tradicionais, razão pela qual, os alunos facilmente associam o jogo físico ao digital.

Porém, quase 95\% dos alunos submetidos ao experimento, manifestaram-se a favor do uso do objeto de aprendizagem N'SAMAT durante as aulas de matemática. Mas, por outro lado, para 5\% alunos essa visão diferenciada de ensino não foi acolhida e para eles a forma tradicional de ensino é melhor. No entanto, percebemos pelos resultados, que a aprendizagem destes alunos por meio do objeto de aprendizagem N'SAMAT melhorou. Dos 95\% a favor do uso do objeto de aprendizagem, $60 \%$ começaram a agir de forma mais autônoma, estabelecendo relações que até então, antes do uso do N'SAMAT, não tinham compreendido. Associado a Teoria de Aprendizagem Significativa de Ausubel, sugere-se que os alunos realizem aprendizagens significativas por si próprios, o que é o mesmo que aprender a aprender. Assim, garantem a compreensão e a facilitação de novas aprendizagens ao ter-se um suporte básico na estrutura cognitiva prévia construída pelo sujeito. Os alunos ao se encontrarem diante de uma situação em que tiveram que desenvolver um pensamento crítico, apresentam maior envolvimento, interesse e dedicação, eles passaram a enxergar a Aritmética em seu dia a dia de uma forma prática e objetiva, não apenas a aritmética vista nos manuais didáticos sem vida e muito longe da sua realidade.

\section{Considerações finais}

O artigo coloca em evidência os benefícios da integração de objeto de aprendizagem no ensino básico em Moçambique. Este mecanismo foi se desenrolado sobre uma série de propostas tendo em vista o atual estágio da integração das tecnologias e dos jogos tradicionais na educação em Moçambique.

O Objeto de aprendizagem N'SAMAT, desenvolvido a partir do reaproveitamento de jogo tradicional com aspectos socioculturais muito fortes, é capaz de contribuir para aprendizagem significativa do aluno no ensino básico, de acordo com padrões sociais, culturais e tecnológicos. Contudo, os resultados apresentados, mostram que a metodologia aplicada para testar o uso do objeto de aprendizagem N'SAMAT, apresentou variabilidade de aproveitamento pedagógico ao nível da significância de 95\% aproximadamente, logo indica que o N'SAMAT possui potencial de aprendizagem. Para tal constatou que: 
- Os alunos da zona urbana têm nota média superior aos alunos da zona rural, independentemente do tempo de exposição ao jogo;

- Os dados evidenciam que existe efeito da exposição ao jogo (pré-teste e pós-teste) sobre o desempenho médio de todos os alunos, independente de zona de origem, de sexo e da idade com Valor $\mathrm{p}<0,0001$;

- A nota média dos alunos após a exposição ao jogo é de 12,1 valores sendo significativamente maior que a nota média dos alunos antes da exposição ao jogo que é 7,0 valores. Este aumento médio da nota (aproximadamente 5 pontos em média) ocorreu nas duas zonas de interesse, isto é, tanto na zona urbana como na zona rural.

\section{Referências}

AUSUBEL, D. P. Educational psychology: a cognitive view. New York: Holt Rinehart \& Winston, 1968.

AUSUBEL, D. P.; NOVAK, J. D.; HANESIAN, J. Psicologia educacional. Rio de Janeiro: Interamericana, 1980.

BEZERRA, M. G.; KASSOUF, Ana Lúcia. Análise de fatores que afetam o desempenho escolar nas escolas das áreas urbanas e rurais do Brasil. Anais. Fortaleza: SOBER, 2006.

CASTRO, M.H.G. de. As desigualdades regionais no sistema educacional brasileiro. In: HENRIQUES, R. Desigualdade e pobreza no Brasil. Rio de Janeiro: IPEA, 2000. p. 425-458.

FIORENTINI, D. Alguns modos de ver e conceber o ensino da matemática no Brasil. Revista Zetetiké, Campinas, v. 3, n. 4, p. 01 - 37, 1995.

GOLIAS, M. EDUCAÇÃO BÁSICA: Temáticas e Conceitos. Moçambique: DINAME, Editora Escolar, S/E, 1999.

INDE/MINED; Plano Curricular do Ensino Básico. Moçambique: Edição: (C INDE/MINED, Nº de registo: 4132/RLINLD /2003

MINISTÉRIO DA EDUCAÇÃO DE MOÇAMBIQUE, Plano Estratégico da Educação 2012-2016: Construindo competências para um Moçambique em constante desenvolvimento. Maputo Moçambique: 12 de junho, 2012; 2012.

MOREIRA, M. A. Aprendizagem significativa: da visão clássica à visão crítica, 2006. Disponível em: <http://www.if.ufrgs.br/ moreira/visaoclasicavisaocritica.pdf> Acesso em: 04 abr. 2016.

MOREIRA, M. A. \& MASINI, E. A. F. S. Aprendizagem significativa: a teoria de David Ausubel. $2^{\text {a }}$ ed. São Paulo, Centauro, 2006.

NUNES, J. M. V., Almouloud, S. A., \& Guerra, R. B. O Contexto da História da Matemática como Organizador Prévio. Bolema 23 (35B), 537-561, 2010.

RONCA, A. C. C. O. Efeito de organizadores prévios na aprendizagem significativa de textos didáticos. Dissertação de Mestrado. São Paulo, Pontifícia Universidade Católica, 1976.

SAS on Demand for Academics. Disponível em: <https://www.sas.com/en_us/software/on-demandfor-academics.html>. Acessado em 13 de março de 2018.

TAROUCO, L. M. R. (Org.). Objetos de Aprendizagem: Teoria e prática. $1^{\text {a }}$ Edição. Porto Alegre - RS: Editora EVAGRAF LTDA, 2014.

VASCONCELOS, R. N.; LIMA, Ivoneide Pinheiro de. Formação inicial de Professor de Matemática: memória e perspectiva. In: BARRETO, Marcília Chagas; PINHEIRO, Joserlene Lima; CARVALHO, Rodrigo Lacerda; MAIA, Dennys Leite (ORG). (Org.). Matemática, Aprendizagem e Ensino. 1ed. Fortaleza: EdUECE, 2013, v. 1, p. 77-92. 